Documentation et bibliothèques

\title{
Construction et numérisation : la construction de grandes bibliothèques reste à l'ordre du jour
}

\section{Gaston Bernier}

Volume 47, numéro 3, juillet-septembre 2001

URI : https://id.erudit.org/iderudit/1032577ar

DOI : https://doi.org/10.7202/1032577ar

Aller au sommaire du numéro

Éditeur(s)

Association pour l'avancement des sciences et des techniques de la

documentation (ASTED)

ISSN

0315-2340 (imprimé)

2291-8949 (numérique)

Découvrir la revue

Citer ce document

Bernier, G. (2001). Construction et numérisation : la construction de grandes bibliothèques reste à l'ordre du jour. Documentation et bibliothèques, 47(3),

91-92. https://doi.org/10.7202/1032577ar

Tous droits réservés (c) Association pour l'avancement des sciences et des techniques de la documentation (ASTED), 2001
Ce document est protégé par la loi sur le droit d'auteur. L'utilisation des services d'Érudit (y compris la reproduction) est assujettie à sa politique d'utilisation que vous pouvez consulter en ligne.

https://apropos.erudit.org/fr/usagers/politique-dutilisation/ 


\title{
Construction et numérisation : la construction de grandes bibliothèques reste à l'ordre du jour
}

\begin{abstract}
Les livres sont d'implacables envahisseurs. Mine de rien, avec une patience infinie et toujours plus nombreux, ils se rendent maître des lieux. Ils ont tôt fait de déborder des bibliothèques où ils sont assignés à résidence [...]

[...]

Au fil du temps, les livres sont devenus de féroces colonisateurs. Ils bouffent sans cesse de l'espace; [...] La vraie ambition des livres est de chasser les hommes des bibliothèques [...] et d'en occuper tout le territoire pour une grandiose et solitaire jouissance.
\end{abstract}

Bernard Pivot, Le métier de lire; réponses à Pierre Nora, Paris : Gallimard, 1990, 2001. p. 220-221. (Folio 3552)

On aurait pu croire que la numérisation de la documentation, la mise sur pied de banques de données électroniques, bibliographiques ou textuelles, la généralisation des cédéroms et la consultation à distance auraient eu un impact négatif sur les grands projets de construction de bibliothèques. Logiquement, il serait normal que les bâtisses perdent de leur importance face à l'évolution observée: le stock de documents, son volume tout au moins, devrait cesser d'exercer une pression imparable sur les locaux trop peu nombreux et sur un espace presque toujours limité. La miniaturisation et l'informatisation, de concert avec la multiplication des ordinateurs domestiques, devraient marquer l'avenir et les besoins documentaires. Continuera-t-on de visiter les établissements documentaires autant que par le passé si on dispose d'un ordinateur chez soi, si on a les données ou les textes désirés à portée de doigt? On s'attendrait également à ce qu'un autre facteur, les pratiques de rationalisation des collections imprimées, influence aussi les calculs de surface de plancher nécessaire au logement des titres tombant dans l'orbe des bibliothèques « rationalisées ». Enfin, on pourrait croire que la production imprimée devrait sinon diminuer, du moins ne pas dépasser le plateau actuel, si les hérauts de la société sans papier ne se sont pas trompés. Bref, à vue de nez, on pourrait entrevoir la mise en place de nouveaux paradigmes.

Pourtant, la fièvre des constructions semble se prolonger, attire l'opinion pu- blique et revient à l'ordre du jour. Sans toutefois offrir une photo d'ensemble du phénomène, Documentation et bibliothèques a publié, il y a quelques mois (juilletseptembre 2000), un article de YvonAndré Lacroix sur de nouvelles bibliothèques publiques françaises. La première phrase était «La France connaît une explosion sans précédent de son parc immobilier de bibliothèques municipales ». Dans la présente livraison, on trouvera deux articles primés par la Grande bibliothèque du Québec et portant sur les motifs sociaux et les justifications à l'appui de nouveaux bâtiments à fonction documentaire.

L'un est signé par la lauréate du prix GBQ-McGill, Caroline Fodor. L'auteur se pose d'entrée de jeu la question essentielle: «A-t-on réellement besoin de grandes bibliothèques? - Bien sûr que oui, répond-elle ". Les bibliothèques deviennent des lieux d'échanges ou des agoras. Elles héritent de nouvelles responsabilités. Au demeurant, il est toujours plus facile de s'appuyer sur du neuf que d'essayer, souvent péniblement, de rafistoler du vieux. Enfin, l'auteur signale, à juste titre, le rôle symbolique de la bibliothèque.

De son côté, la gagnante du prix GBQ-Marie-Claire Daveluy, Geneviève Gamache Vaillancourt, insiste sur le fait que la bibliothèque virtuelle n'est pas encore à portée de main, que les bibliothèques doivent maintenir un équilibre entre l'actuel et l'inactuel, entre ce qui est contemporain et ce qui est du passé, et elle présente la bibliothèque comme un instrument de démocratisation - à l'égal d'Internet pour ses zélateurs. Madame Gamache présente tour à tour les missions symbolique, sociale et institutionnelle des bibliothèques.

Les deux autres articles de la livraison peuvent sembler, à première vue, sans liens avec le filon des constructions. Et pourtant! Philippe Sauvageau, actuel directeur de la Bibliothèque de l'Assemblée nationale, bien connu ici et ailleurs pour ses talents de «constructeur», présente aux lecteurs de la revue le programme des centres de lecture et d'animation culturelles (CLAC) de l'Agence de coopération culturelle et technique: sa naissance, ses objectifs, les crédits alloués, ses réalisations et ses perspectives. II n'est pas question dans l'article de bâtiments à construire ou de locaux à rénover. Mais le thème transparaît à l'occasion. De fait, le logement des bibliothèques mises sur pied relève des autorités locales. Monsieur Sauvageau participe au programme depuis sa mise sur pied dans les années 1980. Aussi est-il on ne peut mieux placé pour le présenter au monde québécois et international de la documentation.

Le dernier article de l'actuel numéro est consacré à la révolution numérique en cartographie. Son sous-titre porte «la bibliothèque à l'ère électronique ». L'auteur, Jean-François Palomino de la Bibliothèque nationale, note que les modes de consultation, de diffusion et de conservation dans le domaine de la cartographie changent rapidement. II souligne que la 
production de cartes imprimées est en baisse. On peut se demander si le phénomène relevé est une exception ou si on peut l'observer également dans l'ensemble des secteurs documentaires.

Somme toute, le thème abordé est des plus passionnants à l'heure où l'État québécois lance le chantier de la Grande bibliothèque, son troisième dans le domaine depuis 1910, après celui de la $\mathrm{Bi}$ bliothèque de l'Assemblée nationale et après la rénovation de l'édifice Holt de la Nationale du Québec. Tout n'est pas dit et fixé. II faudra observer les tendances légères autant que les lourdes. On peut croire que la question des locaux, des constructions, des rénovations, de l'espace nécessaire, des avantages comparés de la proximité et des grandes bibliothèques continuera de retenir l'attention.

\section{Gaston Bernier}

